\title{
SAR IMAGE DENOISING BASED ON MULTI-SCALE WEIGHTED NUCLEAR NORM MINIMIZATION
}

\author{
Yanling An ${ }^{a}$, Jiaxin $\mathrm{Li}^{a}$, Qi Zhanga,b, Xiaole $\mathrm{Ma}^{\mathrm{b}}$, \\ Jiao Panga and Shuai Congc

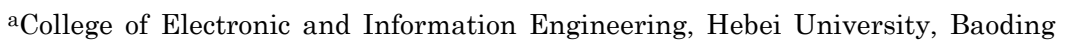 \\ Hebei, P. R. China \\ ${ }^{b}$ College of Computer and Information, Beijing Jiaotong University, Beijing, \\ P. R. China \\ 'Industrial and Commercial College, Hebei University, Baoding, P. R. China
}

\begin{abstract}
Synthetic aperture radar (SAR) is widely used in military and civil fields. The main task of SAR image denoising is to suppress speckle. Combining the advantages of non-subsample shearlet transform (NSST) and weighted nuclear norm minimization (WNNM), we propose a SAR image denoising algorithm based on multi-scale weighted nuclear norm minimization. Firstly, speckle is transformed into additive noise by logarithmic transform. Secondly, NSST is used to decompose the image to low and high sub-bands coefficients, and we can denoise NSST coefficients by WNNM. Finally, NSST is used to reconstruct denoised images. The experimental results show that the proposed algorithm retains the local structure of the image better and achieves a good visual effect.
\end{abstract}

\footnotetext{
${ }^{*}$ Corresponding author.

E-mail address: e-mail: cvmdsp@163.com (Shuai Cong).
}

Copyright ( 2019 Scientific Advances Publishers

2010 Mathematics Subject Classification: 80.

Submitted by Jianqiang Gao.

Received June 25, 2019 
Keywords: SAR image denoising, non-subsample shearlet transform, weighted nuclear norm minimization.

\section{Introduction}

SAR is an active microwave remote sensor that can generate high resolution images. Due to its advantages of real-time observation of the ground at any time, multi-band, penetrating power, and high spatial resolution, it has received more and more attention from various countries and has been widely used in many fields. However, due to its coherent imaging mechanism, SAR images contains speckle noise, which will give a bad effect on the image post-processing. In recent years, with the continuous improvement of wavelet transform, it has played a crucial role in the field of SAR image denoising. However, the wavelet basis is usually fixed and cannot fully represent the image. In order to overcome the non-sparseness and lack of directional selectivity of wavelet basis's coefficients in high dimension, scholars have proposed a series of methods for multi-scale geometric analysis of image. Multi-scale decomposition is an effective and practical method for the representation of data characteristics and details. To a certain extent, the characteristics of the data can be more effectively extracted [1]. NSST is a popular multiscale geometric transform. NSST abandons the subsampled mechanism and enhances the directional selectivity and translation invariance of the shearlet transform. It not only has a good ability of capturing the image feature, but also has low computational complexity.

SAR images contain certain spatial structure information. There are a lot of repeated or similar structural content and texture information in many areas of the image. For a given reference block, multiple image blocks similar to it can be found. That is, it has non-local similarity. The matrix composed of these image blocks is considered to be low-rank [2,3]. The low-rank matrix contains a lot of redundant information that can be 
used to recover the missing data. Candes and Recht prove that most low rank matrix problems can be minimized by nuclear norm minimization (NNM) [4]. In the NNM algorithm, the weights of all singular values are given the same value, so that the algorithm cannot shrink according to the importance of singular values when denoising, resulting in the prior information of the image cannot be fully utilized. In order to make full use of the prior information of the image, Gu et al. [5] proposed weighted nuclear norm minimization (WNNM). WNNM gives different weights to different singular values, which utilizes the prior information of the image effectively, and improves the flexibility of the nuclear norm. This paper uses the algorithm based on multi-scale weighted nuclear norm minimization for SAR image denoising, which effectively reduces the speckle noise, and maintains the texture and edge of the image.

\section{Basic Theories}

\subsection{Speckle model}

In general, speckle noise is fully developed [6, 7]. Goodman proved that the fully developed speckle is a kind of multiplicative noise, and its model is

$$
I(x, y)=R(x, y) \times F(x, y),
$$

where $(x, y)$ is the coordinate of the center pixel of the resolution cell, $I(x, y)$ is the strength of the image contaminated by speckle, $R(x, y)$ is the real image that should be observed, and $F(x, y)$ is the speckle noise. $F(x, y)$ and $R(x, y)$ are independent of each other, and $F(x, y)$ is a second-order stable gamma distribution with an average of 1 .

For the convenience of de-noising processing, the logarithmic transform is performed on both sides of Equation (1) to turn the multiplicative noise into additive noise. The equation is as follows:

$$
\lg (I(x, y))=\lg (R(x, y))+\lg (F(x, y)) .
$$


After the logarithmic transform, $\lg (F(x, y))$ conforms to the Gaussian distribution. But the noise at this time is not Gaussian white noise, so general noise removal methods for Gaussian white noise cannot be used.

\subsection{Multiscale geometric transformation}

NSST is an extension of sheatlet. The NSST transformation is divided into two parts: multi-scale transformation and multi-direction transformation. Multi-scale transformation uses non-sampling pyramids to decompose the image. By $k$-level decomposition of the image, one lowfrequency micro-image and $k$ high-frequency micro-images can be obtained. These $k+1$ sub-image are the same size as the source images. Multi-directional transformation maps the standard shearlet filter from a pseudo-polarized network system to a Cartesian coordinate system, no further sampling, which improves redundancy, and overcomes the disadvantages of translational variability. NSST not only greatly reduces noise, but also preserves more useful original image information. NSST overcomes the disadvantage that wavelet cannot optimally represent image and curvelet is difficult to discretization. At the same time, it solves the problem that the contourlet transform does not conform to the multi-resolution multiple analysis theory, and the implementation of NSST is simpler and more flexible.

\subsection{Weighted nuclear norm minimization}

In actual SAR image processing, the data matrix of the original image is often low rank or approximately low rank, but the presence of speckle undermines this low rank characteristic. In order to restore the low rank effect of the original image, NNM performs matrix approximation by solving the minimum nuclear norm of the matrix [5]. From the soft threshold of singular values, NNM can effectively maintain the details and low rank structure of the image. However, NNM gives the same weight to all singular values, so that all singular values shrink the same level and cannot identify singular values which contains important information. Therefore, we use WNNM algorithm to denoise SAR images. 
The formula (2) can be simple to

$$
y=x+n,
$$

where $y$ is the noise image, $x$ is the clean image, and $n$ is the Gaussian noise with a variance of $\sigma_{n}$. First, $y$ is divided into several blocks, where the $j$-th block is $y_{j}$. Then the method of block matching is used to find similar blocks in the search window, and these non-local similar blocks are aggregated to form a block matrix $Y_{j}$. In this way, the formula (3) can be converted to

$$
Y_{j}=X_{j}+N_{j}
$$

where $X_{j}$ and $N_{j}$ are the block matrices of the original image and noise, respectively. Since $X_{j}$ is low-rank, it can be repaired with WNNM. The solution is as follows:

$$
\hat{X}_{j}=\arg \min _{x_{j}} \frac{1}{\sigma_{n}^{2}}\left\|Y_{j}-X_{j}\right\|_{F}^{2}+\left\|X_{j}\right\|_{\omega, *},
$$

where $\left\|X_{j}\right\|_{\omega},{ }^{*}=\sum_{i}\left|\omega_{i} \mu_{i}\left(X_{j}\right)\right|_{1}, \omega=\left[\omega_{1}, \cdots, \omega_{n}\right], \omega_{i} \geq 0 . \omega_{i}$ is the nonnegative weight assigned to $\mu_{i} \cdot\|\|_{F}$ is the $F$ norm. In general, larger singular values carry the principal component energy in the SAR image. Therefore, during the SAR image denoising, the larger the singular value, the smaller the contraction. And the smaller the singular value, the larger the contraction. Combined with threshold contraction, we can get

$$
\hat{X}=U S_{\omega}(\Sigma) V^{T}
$$

where $S_{\omega}(\Sigma)$ represents the soft threshold function of the matrix $\sum$ under the weight $\omega$. 
In general, the singular value is non-descending, so the weight should also be non-descending. The solution of the WNNM can converge to a fixed point when the weight is non-descending. WNNM is used to denoise on each image block, and then all the blocks are gathered together to obtain the denoised image $X$.

\section{SAR Image Denoising Based on Multi-Scale Weighted Nuclear Norm Minimization}

In this paper, NSST and WNNM are combined to denoise SAR images. The denoising steps can be described as following.

Step 1. This paper chooses logarithmic transform to preprocess the image and the model of speckle noise in the SAR image can be turn into additive noise. Next, we denoise the image.

Step 2. The logarithmic SAR image is decomposed at multiple scales to produce a low frequency sub-band and two high frequency sub-bands by NSST.

Step 3. WNNM is used to denoise each scales of NSST coefficients. The first step is to pre-calculate the adjacent block index of the selected block in the search window based on these parameters. The second step estimates the local noise level of each block, and the noise level changes with the number of non-local blocks. The lower the noise level, the less the number of non-local blocks. The third step is to perform block matching and calculate the effective similar blocks of each block. The fourth step is to obtain the decomposed singular value according to formula (6) and get the block estimate of all blocks. The fifth step is to aggregate image blocks and return to the second step for iterative correction until it meets the requirements.

Step 4. The image of each layer is reconstructed by inverse NSST, and the clean image is finally obtained. 


\section{Experimental Results and Analysis}

To verify the feasibility of the proposed algorithm in this paper, we compare the proposed algorithm (NSST-WNNM) with the following SAR image denoising algorithms: SAR image de-noising based on GNL-means with optimized pixel-wise weighting in non-subsample shearlet domain [8] (GNL-NSST), Bayesian shearlet shrinkage for SAR image de-noising via sparse representation [9] (BSSR), and SAR image denoising via sparse representation in shearlet domain based on continuous cycle spinning [10] (CS-BSSR).

Two SAR images taken by TerraSar-X are compared experimentally in this chapter, as shown in Figure 1.

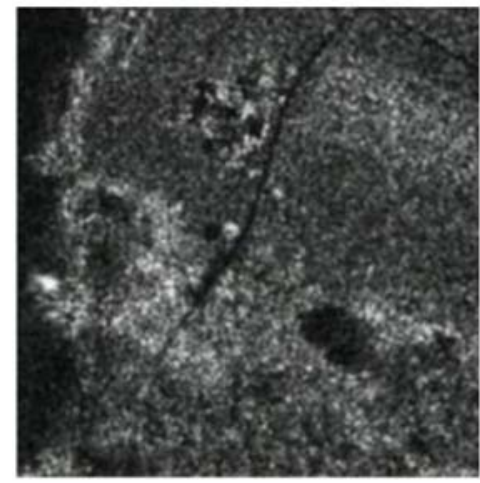

(a)

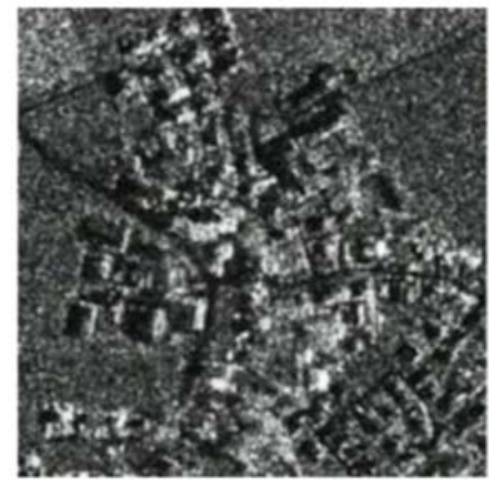

(b)

Figure 1. Real SAR image: (a) woods SAR image, and (b) field SAR image.

Figure 1(a) shows an SAR image of the woods and Figure 1(b) shows an SAR image of the field. The experimental results obtained by applying the denoising methods above are shown in Figures 2 and 3. 


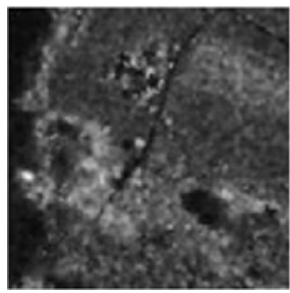

(a)

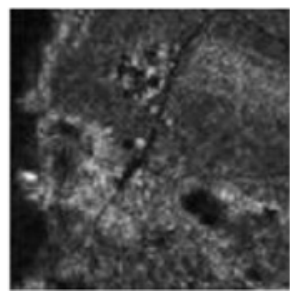

(b)

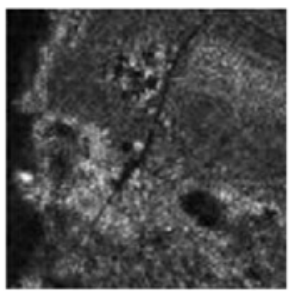

(c)

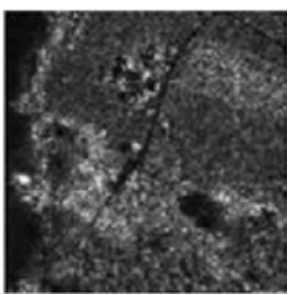

(d)

Figure 2. SAR images of the woods after denoised using different denoising methods: (a) BSSR, (b) GNL-NSST, (c) CS-BSSR, and (d) NSST-WNNM.

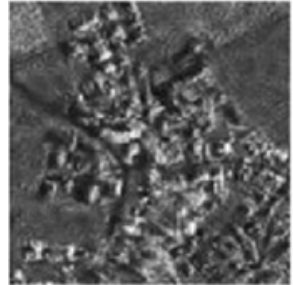

(a)

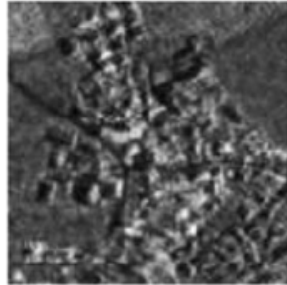

(b)

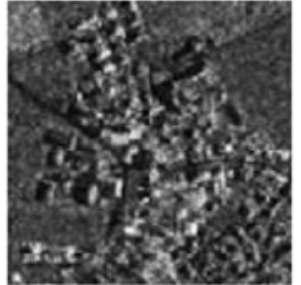

(c)

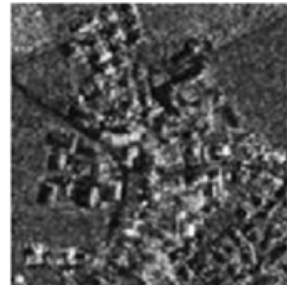

(d)

Figure 3. SAR images of the field after denoised using different denoising methods: (a) BSSR, (b) GNL-NSST, (c) CS-BSSR, and (d) NSST-WNNM.

From Figures 2 and 3, it can be seen that the edges of the image after the BSSR method for noise reduction are not clear. The GNL-NSST method was used to remove part of the texture of the denoised image, which caused the details to be lost and the whole image was blurred and distorted, seriously affecting the visual effects. Artificial texture is added to the denoised image by CS-BSSR method. It can be seen that the local maintenance ability of NSST-WNNM is better, the texture details and edge information can be better expressed, and the visual effect is better than other methods.

In order to more intuitively display the denoising effect of each denoising method, this paper lists several image quality evaluation indicators, including: peak signal to noise ratio (PSNR), which is used to 
measure the denoising ability of the algorithm; equivalence number of looks (ENL), which is used to measure the visual effect of the image after denoising; edge representation index (EPI), which is used to measure the details that the algorithm can retain. The larger the ENL value, the better the visual effect. The closer the EPI value is to 1 , the stronger the ability to maintain details. Table 1 gives a comparison of the denoising algorithms.

Table 1. The evaluation indicators of SAR image denoising methods

\begin{tabular}{|c|c|c|c|c|c|c|}
\hline & \multicolumn{3}{|c|}{ SAR image of the woods } & \multicolumn{3}{c|}{ SAR image of the field } \\
\hline Denoise algorithm & PNSR & ENL & EPI & PNSR & ENL & EPI \\
\hline BSSR & 33.11 & 22.45 & 0.90 & 31.55 & 15.56 & 0.88 \\
\hline GNL-NSST & 36.67 & 25.55 & 0.98 & 35.01 & 20.65 & 0.95 \\
\hline CS-BSSR & 35.85 & 24.19 & 0.97 & 34.33 & 19.90 & 0.94 \\
\hline NSST-WNNM & 37.07 & 25.95 & 0.99 & 35.48 & 21.12 & 0.97 \\
\hline
\end{tabular}

As shown in Table 1, NSST-WNNM algorithm has the highest PSNR and ENL, and EPI is closest to 1, indicating that this algorithm has the strongest ability on maintaining the details and denoising. From the experimental results, we can see that the algorithm proposed in this paper has good results in detail retention and noise removal, which proves its feasibility and effectiveness.

\section{Summary}

This article briefly introduces the basic theories of WNNM and NSST. Based on the advantages of the two methods, the SAR image denoising based on multi-scale weighted nuclear norm minimization is proposed. By comparing with other denoising algorithms, it can be seen that the proposed algorithm can be applied to different types of SAR images. It can effectively suppress speckle noise, better preserve the local structures such as edges and textures, produce less edge defects, and has better denoising effect. This paper will focus on improving the efficiency of computing similar blocks and the WNNM parameter adaptability in the future. 


\section{Acknowledgements}

This work was supported in part by National Natural Science Foundation of China under Grant 61572063 and 61401308, Opening Foundation of Machine Vision Engineering Research Center of Hebei Province under Grant 2018HBMV02, Science Research Project of Hebei Province under Grant QN2016085, and Natural Science Foundation of Hebei University under Grant 2014-303.

\section{References}

[1] Shuaiqi Liu, Mingzhu Shi, Zhihui Zhu and Jie Zhao, Image fusion based on complexshearlet domain with guided filtering, Multidimensional Systems and Signal Processing 28(1) (2017), 207-224.

DOI: https://doi.org/10.1007/s11045-015-0343-6

[2] S. Liu, Q. Hu, T. Liu and J. Zhao, Review on synthetic aperture radar image denoising algorithms, Journal of Ordnance Equipment Engineering 39(12) (2018), 106-112, 252.

[3] Shuaiqi Liu, Qi Hu, Pengfei Li, Jie Xhao, Ming Liu and Zhihui Zhu, Speckle suppression based on weighted nuclear norm minimization and grey theory, IEEE Transactions on Geoscience and Remote Sensing 57(5) (2019), 2700-2708.

DOI: https://doi.org/10.1109/TGRS.2018.2876339

[4] Shuaiqi Liu, Tao Zhang, Hailiang Li, Jie Zhao and Huiya Li, Medical image fusion based on nuclear norm minimization, International Journal of Imaging Systems and Technology 25(4) (2015), 310-316.

DOI: https://doi.org/10.1002/ima.22145

[5] Shuhang Gu, Lei Zhang, Wangmeng Zuo and Xiangchu Feng, Weighted Nuclear Norm Minimization with Application to Image Denoising, 2014 IEEE Conference on Computer Vision and Pattern Recognition (CVPR), IEEE Computer Society, 2014.

DOI: https://doi.org/10.1109/CVPR.2014.366

[6] Shuaiqi Liu, Mingzhu Shi, Shaohai Hu and Yang Xiao, Synthetic aperture radar image de-noising based on shearlet transform using the context-based model, Physical Communication 13(Part C) (2014), 221-229.

DOI: https://doi.org/10.1016/j.phycom.2014.02.002

[7] Shuaiqi Liu, Tong Liu, Lele Gao, Hailiang Li, Qi Hu, Jie Zhao and Chong Wang, Convolutional neural network and guided filtering for SAR image denoising, Remote Sensing 11(6) (2019); Article 702.

DOI: https://doi.org/10.3390/rs11060702 
[8] Shuaiqi Liu, Yu Zhang, Qi Hu, Ming Liu and Jie Zhao, SAR image de-noising based on GNL-means with optimized pixel-wise weighting in non-subsample shearlet domain, Computer and Information Science 10(1) (2017), 16-22.

DOI: https://doi.org/10.5539/cis.v10n1p16

[9] Shuaiqi Liu, Shao Hai Hu, Yang Xiao and Yongli An, Bayesian shearlet shrinkage for SAR image de-noising via sparse representation, Multidimensional Systems and Signal Processing 25(4) (2014), 683-701.

DOI: https://doi.org/10.1007/s11045-013-0225-8

[10] Shuaiqi Liu, Ming Liu, Peifei Li, Jie Zhao, Zhihui Zhu and Xuehu Wang, SAR image denoising via sparse representation in shearlet domain based on continuous cycle spinning, IEEE Transactions on Geoscience and Remote Sensing 55(5) (2017), 2985-2992.

DOI: https://doi.org/10.1109/TGRS.2017.2657602 